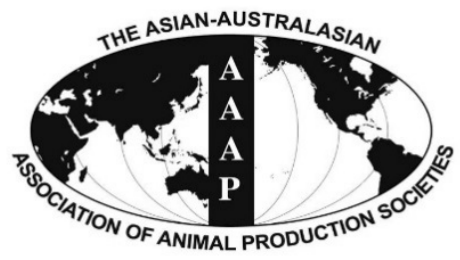

Open Access

Asian Australas. J. Anim. Sci.

Vol. 29, No. 11 : 1576-1584 November 2016

http://dx.doi.org/10.5713/ajas.16.0336

www.ajas.info

pISSN 1011-2367 elSSN 1976-5517

\title{
Characterization of Heterologously Expressed Acetyl Xylan Esterase1 Isolated from the Anaerobic Rumen Fungus Neocallimastix frontalis PMA02
}

\author{
Mi Kwon ${ }^{2,3}$, Jaeyong Song ${ }^{1,4}$, Hong-Seog Park ${ }^{5}$, Hyunjin Park ${ }^{1}$, and Jongsoo Chang ${ }^{1, *}$ \\ ${ }^{1}$ Department of Agricultural Science, Korea National Open University, Seoul 03087, Korea
}

\begin{abstract}
Acetyl xylan esterase (AXE), which hydrolyzes the ester linkages of the naturally acetylated xylan and thus known to have an important role for hemicellulose degradation, was isolated from the anaerobic rumen fungus Neocallimastix frontatlis PMA02, heterologously expressed in Escherichi coli (E.coli) and characterized. The full-length cDNA encoding NfAXE1 was 1,494 bp, of which $978 \mathrm{bp}$ constituted an open reading frame. The estimated molecular weight of NfAXE1 was $36.5 \mathrm{kDa}$ with 326 amino acid residues, and the calculated isoelectric point was 4.54 . The secondary protein structure was predicted to consist of nine $\alpha$-helixes and $12 \beta$-strands. The enzyme expressed in E.coli had the highest activity at $40^{\circ} \mathrm{C}$ and $\mathrm{pH}$. The purified recombinant NfAXE1 had a specific activity of 100.1 $\mathrm{U} / \mathrm{mg}$ when $p$-nitrophenyl acetate $\left(p\right.$-NA) was used as a substrate at $40^{\circ} \mathrm{C}$, optimum temperature. The amount of liberated acetic acids were the highest and the lowest when $p$-NA and acetylated birchwood xylan were used as substrates, respectively. The amount of xylose released from acetylated birchwod xylan was increased by 1.4 fold when NfAXE1 was mixed with xylanase in a reaction cocktail, implying a synergistic effect of NfAXE1 with xylanase on hemicellulose degradation. (Key Words: Acetyl Xylan Esterase, Neocallimastix frontalis, Hemicellulose, Anaerobic Rumen Fungus)
\end{abstract}

\section{INTRODUCTION}

Hemicellulose is the second-most abundant structural carbohydrate and accounts for approximately $20 \%$ to $30 \%$ of the dry weight of the plant cell wall. The main component of its structural backbone is xylan which consists of $\beta-1,4-$ linked xylopyranosyl residues with various substituted side groups, such as arabinose and $O$-acetyl, and ferulic (4hydroxy-3-methoxycinnamic), $p$-coumaric, $L$-arbinofuranosyl, and 4-O-methylglucuronyl residues (Thomson, 1993). In several hardwood species, about $60 \%$ to $70 \%$ of xylose residues are esterified with acetic acid, and up to $2.7 \%$ of the dry matter of grasses consists of $O$-acetyl groups linked to

\footnotetext{
* Corresponding Author: Jongsoo Chang. Tel: +82-2-3668-4636, Fax: +82-2-3673-2381, E-mail: jschang@knou.ac.kr

${ }^{2}$ InfoBoss Incorporation, Seoul 07766, Korea.

${ }^{3}$ Institute of Biological Chemistry, Washington State University, Pullman, WA 99163, USA.

${ }^{4}$ Department of Animal Sciences, Kyungpook National University, Sangju 37224, Korea.

${ }^{5}$ GnCBio Incorporation, Daejeon 34069, Korea.

Submitted Apr. 27, 2016; Revised May 29, 2016; Accepted Jun. 9, 2016
}

the C-2 or C-3 of the xylopyranose residues (Thomson, 1993). The presence of the acetyl group in the xylan backbone hinders the complete degradation of hemicellulose; consequently, the removal of the acetyl group is a very important step in many industrial applications which utilize plant biomass, including biofuel production systems (Biely, 1985).

Acetyl xylan esterase (AXE), an enzyme able to hydrolyze ester linkages at position C-2 and/or C-3 of the acetyl groups in xylose moieties of naturally acetylated xylans, was first isolated from a crude culture of the filamentous fungus Schizophyllum commune (Biely, 1985). Different AXEs have subsequently been purified and characterized from many xylanolytic fungi, including Penicillium purpurogenum (Gutiérrez et al., 1998), Aspergillus oryzae, and Trichoderma reesei (Tenkanen, 1998), as well as from a rumen bacterium Fibrobacter succinogenes S85 (McDermid et al., 1990). Coutinho and Henrissat (1999) classified carbohydrate esterases (CE) into 14 groups, with AXEs classified into CE families 1 to 7 based on significant sequence diversity. This sequence diversity implies functional diversity, including diverse substrate 
specificity and a distinct regio-selective mode of action (Altaner et al., 2003).

Fibrous carbohydrates can be used as feed resources for herbivores, including non-ruminant herbivores and ruminants. These animals break down the various compounds present in fibrous feed into small molecules through the actions of various carbohydrate-hydrolyzing enzymes secreted by microorganisms present in the rumen, namely, bacteria, protozoa, and fungi-mostly by microbial fermentation. The presence of anaerobic fungi in the rumen was first reported by Orpin (1975). Currently, six genera of anaerobic fungi, namely, Anaeromyces, Caecomyces, Cyllamyces, Neocallimastix, Orpinomyces, and Piromyces are classified in the National Center for Biotechnology Information (NCBI) taxonomy database (http://ncbi.nlm. nih.gov/), most of which have been isolated from either ruminant animals or non-ruminant herbivores (Orpin, 1981). Unlike bacteria or protozoa in the rumen, anaerobic fungi possess rhizoid filaments that penetrate feed particles, resulting in their physical breakdown (Ho et al., 1988). Anaerobic fungi secrete various enzymes, such as endoglucanase (Mountfort and Asher, 1985), $\beta$-glucosidase (Li and Calza, 1991), xylanase and other carbohydratehydrolyzing enzymes (Kwon et al., 2009).

Neocallimastix frontalis ( $N$. frontalis), a genus of obligate anaerobic rumen chytridiomycete fungi in the family Neocallimastigaceae, was first isolated and wellcharacterized by Orpin (1975). It has been known to secrete effective fiber-degrading enzymes with a primary role in the complex microbial ecology of the rumen (Mountfort and Asher, 1985). It is recognized as potent lignocellulose degrader (Wood et al., 1995), thus, regarded as a good genetic resource for lignocellulolytic enzymes that could be used in many industries including bioenergy, refinery, animal production and bioremediation (Kwon et al., 2009). However, isolation and characterization of genes and proteins/enzymes regarding lignocellulose degradation are very limited, mainly because of the difficulties associated with isolation and maintenance of pure stains of anaerobic fungi. Only several enzymes for cellulose and xylan degradation have been characterized. For example, $N$. frontalis $\beta$-D-xylosidase has been purified and characterized biochemically (GarciaCampayo and Wood, 1993) and cellulase fractions rich in endoglucanase activity were separated and biochemically characterized (Wood et al., 1995). Recent genome-wide approaches have provided expressed sequence tag (EST) database and this made possible to isolate functional genes for carbohydrate metabolism including cellulose and xylan degradation (Kwon et al., 2009). Nevertheless, with this enormous amount of nucleotide sequences related to fiber degradation available, only one cDNA clone encoding endo1,4- $\beta$-glucanase from a $N$. frontalis $\mathrm{MCH} 3$ has been heterologously expressed and characterized at the molecular level (Fujino et al., 1998). The aim of this study was to isolate and characterize fiber degradation enzymes from $N$. frontalis.

\section{MATERIALS AND METHODS}

\section{Screening of $\boldsymbol{N} \boldsymbol{A} \boldsymbol{A} \boldsymbol{E} \boldsymbol{1}$ gene and sequence analysis}

The anaerobic rumen fungus $N$. frontalis PMA02 was obtained from the culture collection of the Department of Agricultural Science, Korea National Open University and cultivated in a modified Lowe's medium containing a mixture of $2 \%$ glucose, cellobiose, and starch $(2: 1: 1)$ as carbohydrate sources (Kwon et al., 2009). Escherichi coli (E.coli) clones containing AXE homologs were selected from the constructed EST library (Kwon et al., 2009), and inserts of larger than $1 \mathrm{~kb}$ were first screened by colony polymerase chain reaction (PCR) using $\mathrm{T} 3$ and $\mathrm{T} 7$ primers (Figure 1A). One of the clones (NfAXE1) which contains a putative full-length ORF with a $5^{\prime}$ and a $3^{\prime}$ UTR was selected for further study. The software programs ProtParam

(A)

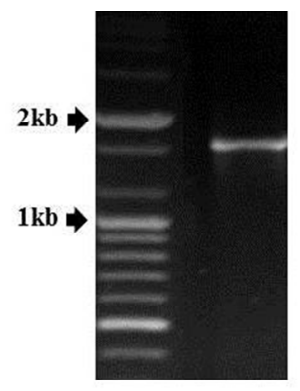

(B)

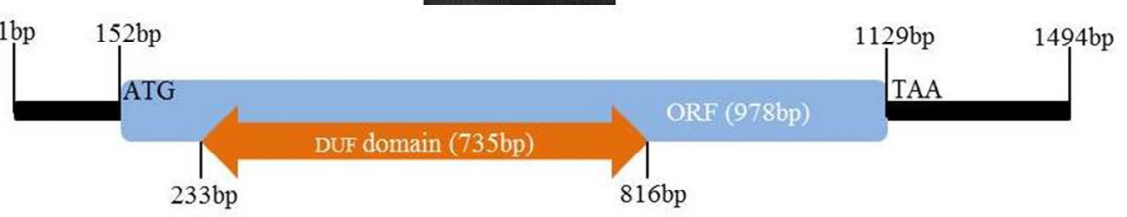

Figure 1. The structure of Neocallimastix frontalis PMA02 acetyl xylan esterase (NfAXE) genomic DNA. (A) DNA electrophoresis results for NfAXE, (B) NfAXE genomic DNA structure. ORF, Open reading frame; DUF, domain of unknown function. 
(http://web.expasy.org/protparam/) and Predict Protein (https://www.predictprotein.org) were used to analyze the physical and chemical parameters of NfAXE1, and SWISSMODEL, a structural bioinformatics web-server (http://swissmodel.expasy.org), was used to determine the tertiary structure of NfAXE1 (Gasteiger et al., 2005).

\section{Recombinant protein expression and purification}

The open reading frame (ORF) with 981 bp was amplified by PCR using a forward (5'-GCEATATG AGAGCCAGTATT ATT-3') and a reverse (5'-CCGGATCC ATTTTCTTCAG CAGAA-3') primers, incorporating the NdeI and BamHI sites (underlined), respectively. The PCR products were then inserted into the NdeI and BamHI restriction sites of the expression vector pET28a (Novagen, Merck KGaA, Darmstadt, Germany) to generate pET28aNfAXE1. The plasmid of pET28a-NfAXE1 was transformed into E.coli BL21 (DE3) cells and cultivated in $50 \mathrm{~mL}$ of lysogeny broth medium containing kanamycin $(50 \mu \mathrm{g} / \mathrm{mL})$ at $37^{\circ} \mathrm{C}$ with shaking at $250 \mathrm{rpm}$. When the optical density of the culture reached 0.5 at $600 \mathrm{~nm}$, expression of the recombinant protein was induced by adding isopropyl- $\beta$-Dthiogalactopyranoside (IPTG) to a final concentration of 1 $\mathrm{mM}$ and decreasing the culture temperature to $25^{\circ} \mathrm{C}$. After a $12 \mathrm{~h}$ incubation, cells were harvested by centrifugation at $2,500 \times \mathrm{g}$ for $5 \mathrm{~min}$ and re-suspended in $8 \mathrm{~mL}$ of native binding buffer (Invitrogen, Carlsbad, CA, USA); following the addition of $8 \mathrm{mg}$ of lysozyme, the cells in suspension were incubated for $30 \mathrm{~min}$ on ice and then ruptured using a US-300 sonicator (Nissei Corp., Osaka, Japan; six 10 s highintensity bursts with a $10 \mathrm{~s}$ cooling period between each burst). The lysates were centrifuged at $3,000 \times \mathrm{g}$ for $15 \mathrm{~min}$ and the supernatants collected. Eight $\mathrm{mL}$ of crude lysate were applied to a Ni-NTA agarose column (Invitrogen, USA) and washed four times with $8 \mathrm{~mL}$ of native washing buffer containing imidazole $(75 \mathrm{mM}, \mathrm{pH} 6.0)$. The protein was eluted with $10 \mathrm{~mL}$ of elution buffer containing imidazole (250 mM, pH 8.0) and collected in $500 \mu \mathrm{L}$ samples.

\section{Sodium dodecyl sulfate-polyacrylamide gel electrophoresis and microfluidic gel electrophoresis}

Protein concentrations were determined by Bradford method using bovine serum albumin as a standard (Bradford, 1976). Crude proteins (109 $\mu \mathrm{g}$ samples) and purified recombinant proteins (3 $\mu \mathrm{g}$ samples) were separated by sodium dodecyl sulfate-polyacrylamide gel electrophoresis (SDS-PAGE) on a $10 \%$ SDS gel. After separation, the gel was stained with Coomassie Brilliant Blue solution containing $10 \%(\mathrm{v} / \mathrm{v})$ phosphoric acid, $10 \%(\mathrm{w} / \mathrm{v})$ ammonium sulfate, $0.12 \%(\mathrm{w} / \mathrm{v})$ Coomassie Brilliant Blue G250, and 20\% $(\mathrm{v} / \mathrm{v})$ methanol. For microfluidic gel electrophoresis, $3 \mu \mathrm{g}$ of each protein sample was loaded to a microfluidic chip and analyzed using the Experion Pro260 automated gel electrophoresis system (Bio-Rad, Hercules, CA, USA) according to the manufacturer's protocol.

\section{Enzyme assay}

The sample with $20 \mu \mathrm{L}$ of volume dissolved in $0.5 \mathrm{M}$ potassium phosphate buffer ( $\mathrm{pH}$ 6.0) was mixed with $0.2 \mu \mathrm{L}$ of protein solution and distilled water to a final total volume of $180 \mu \mathrm{L}$. The reaction was initiated by adding $20 \mu \mathrm{L}$ of 10 $\mathrm{mM}$-NA dissolved in dimethyl sulfoxide and $0.5 \mathrm{M}$ potassium phosphate buffer $(\mathrm{pH}$ 6.0). After a 15 min incubation at $40^{\circ} \mathrm{C}$, the amount of $p$-NA released was estimated by measuring the absorbance at $420 \mathrm{~nm}$ using an automated microplate-based multi-detection reader (FLUOstar Optima; BMG LABTECH, Offenburg, Germany). One enzyme unit was defined as the amount of enzyme which released $1 \mu \mathrm{M}$ of $p$-NA per minute at $40^{\circ} \mathrm{C}$. All assays were carried out at the previously determined optimum reaction temperature and $\mathrm{pH}$.

For the assay using $\alpha$-naphthyl acetate as substrate, 1.34 $\mathrm{mL}$ of $100 \mathrm{mM}$ sodium phosphate buffer $(\mathrm{pH} 7.0), 0.5 \mu \mathrm{L}$ of purified protein solution, and $0.15 \mathrm{~mL}$ of $5 \mathrm{mM} \alpha$-naphthyl acetate dissolved in methanol were incubated together for 15 min at $39^{\circ} \mathrm{C}$, following which the reaction was terminated by adding $1.5 \mathrm{~mL}$ of $10 \%(\mathrm{w} / \mathrm{v})$ SDS containing $0.01 \%(\mathrm{w} / \mathrm{v}) 4^{\prime}-$ amino-2,3'-dimethylazobenzene and the absorbance at 560 $\mathrm{nm}$ measured using an UV-Vis 8453 spectrophotometer (Agilent Technologies, Santa Clara, CA, USA). Alphanaphthol was used as a standard, and one enzyme unit was defined as the amount of enzyme releasing $1 \mu \mathrm{M}$ of $\alpha$ naphthol per minute.

In order to determine the substrate specificity of the purified recombinant protein toward acetylated xylan, birchwood xylan and beechwood xylan were acetylated according to the method of Johnson et al. (1988) using acetic anhydride as an acetyl group donor. First, the purified protein solution was mixed with $100 \mu \mathrm{L}$ of $0.5 \mathrm{M}$ potassium phosphate buffer ( $\mathrm{pH}$ 6.0) and distilled water to a final total volume of $300 \mu \mathrm{L}$. Second, a $200 \mu \mathrm{L}$ sample of $10 \%(\mathrm{w} / \mathrm{v})$ acetylated either birchwood xylan or beechwood xylan was added to the protein solution to initiate the enzymatic reaction. After incubation at $40^{\circ} \mathrm{C}$ for $20 \mathrm{~min}, 10 \mu \mathrm{L}$ of 0.5 $\mathrm{M}$ sulfuric acid was added to terminate enzyme reaction, and the reactions were transferred to microcentrifuge tubes and centrifuged at $8,000 \times g$ for $3 \mathrm{~min}$. The supernatant with $30 \mu \mathrm{L}$ of volume was then injected into an Agilent 1200 HPLC system (Agilent Technologies, USA) equipped with an Agilent 1200 VWD detector (Agilent Technologies, USA) and an Aminex HPX-87 column (Bio-Rad, USA) and separated using $5 \mathrm{mM} \mathrm{H}_{2} \mathrm{SO}_{4}$ as the mobile phase (flow rate $0.6 \mathrm{~mL} / \mathrm{min}$ ) at $25^{\circ} \mathrm{C}$. One enzyme unit was defined as the amount of enzyme releasing $1 \mu \mathrm{M}$ acetic acid per minute. 
For testing the synergistic effect of AXE on xylanase activity, endo-1, 4- $\beta$-xylanase from Trichoderma longibrachiatum (X2629; Sigma, St. Louis, MO, USA) was mixed with recombinant NfAXE1 protein to make a standard solution of $250 \mathrm{U} / \mathrm{mL}$ xylanase and $54 \mathrm{U} / \mathrm{mL}$ NfAXE1. The enzyme mixture with $20 \mu \mathrm{L}$ of volume was mixed with $100 \mu \mathrm{L}$ of $2 \%$ (w/v) acetylated birchwood xylan and incubated at $40^{\circ} \mathrm{C}$ for $30 \mathrm{~min}$, following which $1 \mathrm{ml}$ of Tetrazolium Blue solution [0.05 $\mathrm{M}$ potassium sodium tartrate and $0.005 \%(\mathrm{w} / \mathrm{v})$ Tetrazolium Blue Chloride in $0.05 \mathrm{M} \mathrm{NaOH}$ ] was added and the reaction mixture boiled for $5 \mathrm{~min}$ to terminate the reaction (Jue and Lipke, 1985). Absorbance at $560 \mathrm{~nm}$ was measured using an Agilent UV-Vis 8453 spectrophotometer (Agilent Technologies, USA), with glucose as the standard. One enzyme unit was defined as the amount of enzyme releasing $1 \mu \mathrm{M}$ of reducing sugar per minute at $40^{\circ} \mathrm{C}$.

\section{RESULTS}

\section{Isolation of NfAXE1 clone}

A previously constructed expressed sequence tag (EST) library (Kwon et al., 2009) was used to screen candidate clones containing the AXE homolog of $N$. frontalis PMA02 (NfAXE1). Three contigs and three singletons were identified as putative AXEs, which were subsequently subjected to re-analysis using colony PCR. Among the ESTs in the library, one colony of 1,494 bp insert (Korea Patent Number: 10-098605; Figure 1A) was homologous to an AXE isolated from Orpinomyces sp. PC-2 (AxeA; AF001178.1) and from Neocallimastix patriciarum (bnaI; U66251.1) with
$86 \%$ and $77 \%$ of nucleotide sequence identities, respectively. Sequence analysis of the NfAXE1 exhibited very low identity $(<20 \%)$ with all other AXEs in NCBI database and was deposited in the NCBI database (accession number DI175536.1). Sequence analysis revealed that the selected colony contains an ORF of 978 bp (Figure 1B), a 152 bp 5' untranslated region (UTR), and a 365 bp 3' UTR (Figure 1B), accounting for 1,494 bp of AXE sequence and thereby predicting as a nearly full-length cDNA of $N$. frontalis. This AXE was designated NfAXE1. Sequencing of the genomic DNA sequence encoding NfAXE1 and subsequent comparison with cDNA revealed that NfAXE1 genomic DNA did not have an intron and had only one exon with domain of unknown function (DUF) (Figure 1B).

The estimated molecular weight of NfAXE1 based on its deduced amino acid sequence was 36,505.9 Da with 326 amino acid residues, of which glutamic acid was the most abundant and tryptophan the least abundant (Figure 2). In terms of protein secondary structure, $25.77 \%$ of the amino acids were composed in nine $\alpha$-helixes and $15.95 \%$ in $12 \beta$ strands, with 16 predicted protein binding regions. According to ProtParam analysis results, NfAXE1 was classified as an unstable protein based on its aliphatic index of 86.13 and instability index (II) of 40.03 , leading to an estimated half-life of $20 \mathrm{~h}$ in the yeast cell. The calculated isoelectric point (pI) was 4.54, with 63 negatively charged residues (Asp and Glu) and 38 positively charged residues (Arg, His, and Lys). The existence of highly hydrophobic sequences at the N-terminal region suggested the possible existence of a putative signal peptide with cleavage site

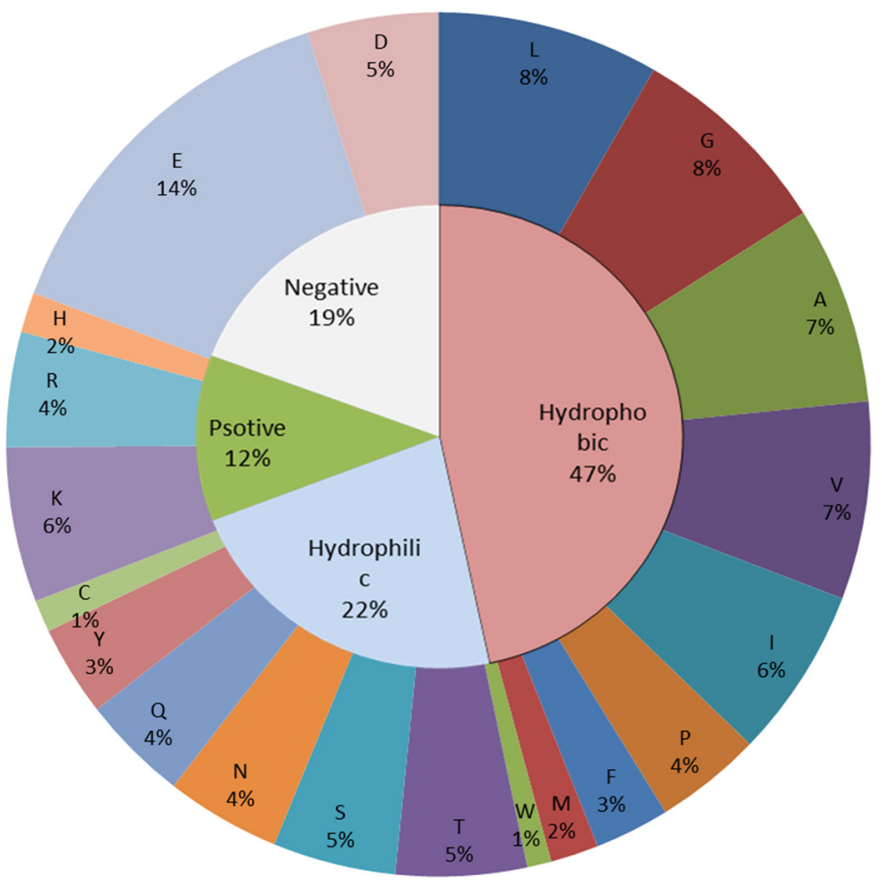

Figure 2. Amino acid composition (outer ring) and amino acid groups (inner ring) of Neocallimastix frontalis PMA02 acetyl xylan esterase (NfAXE1). 
probability 0.559 between residues 22 and 23 .

The deduced amino acid sequence of NfAXE1 cDNA was homologous to AxeA (AAC14690) of Orpinomyces sp. PC-2 and bnaI (AAB69090) of $N$. patriciarum with $70.21 \%$ and $48.74 \%$ similarity, respectively (Figure 3 ). Phylogenetic analysis (Figure 4) revealed that NfAXE1 was located in the same clade with AxeA (AAC14690.1) from the anaerobic rumen fungus Orpinomyces sp. and bnaI (AAB69090.1) from $N$. patriciarum, as well as with WP_014546948.1 from Fibrobacter succinogenes. Partial sequence of the other AXE from $N$. frontalis (AAQ10006.1) showed low sequence similarity $(9.62 \%)$ with NfAXE1. Sequence similarities of AXEs from aerobic fungi, including Tricoderma reesei (CAA93247.1), Aspergillus niger (CAA01634.1), and Fusarium oxysporum (ENH75054.1), were only 2.03\%, $3.24 \%$, and $2.04 \%$, respectively.

Expression, purification and characterization of recombinant NfAXE1

After a $12 \mathrm{~h}$ induction with IPTG, recombinant protein was harvested and purified using a Ni-NTA agarose column
(Figure 5A). During purification, the enzyme activity increased by 101.2 fold, with a yield of $24.4 \%$ and a specific activity of $100.1 \mathrm{U} / \mathrm{mg}$ when $p$-nitrophenyl acetate ( $p$-NA) was used as the substrate (Table 1). Based on the results of microfluidic gel electrophoresis, the final protein product has a purity of $90 \%$ and a molecular mass of $43 \mathrm{kDa}$ (Figure 5B), slightly bigger than predicted native NfAXE1 probably because of $\mathrm{N}$-terminal his-tags. In an enzyme assay using $p$ NA as substrate, the recombinant NfAXE1 achieved peak enzyme activity at $40^{\circ} \mathrm{C}$, with activity subsequently decreasing with increasing incubation temperature (Figure 6A). The enzyme activity of recombinant NfAXE1 increased with increasing $\mathrm{pH}$, reaching peak activity under slightly alkaline conditions (Figure 6B).

Various substrates were tested to determine substrate specificity of the recombinant NfAXE1 under optimum conditions $\left(40^{\circ} \mathrm{C}, \mathrm{pH} 8\right)$. The amount of liberated acetic acid, a marker of AXE activity, was the highest with $p$-NA as substrate and lowest with acetylated birchwood xylan as substrate. When NfAXE1 was mixed with endo-1,4- $\beta$ xylanase from Trichoderma longibrachiatum, the amount of

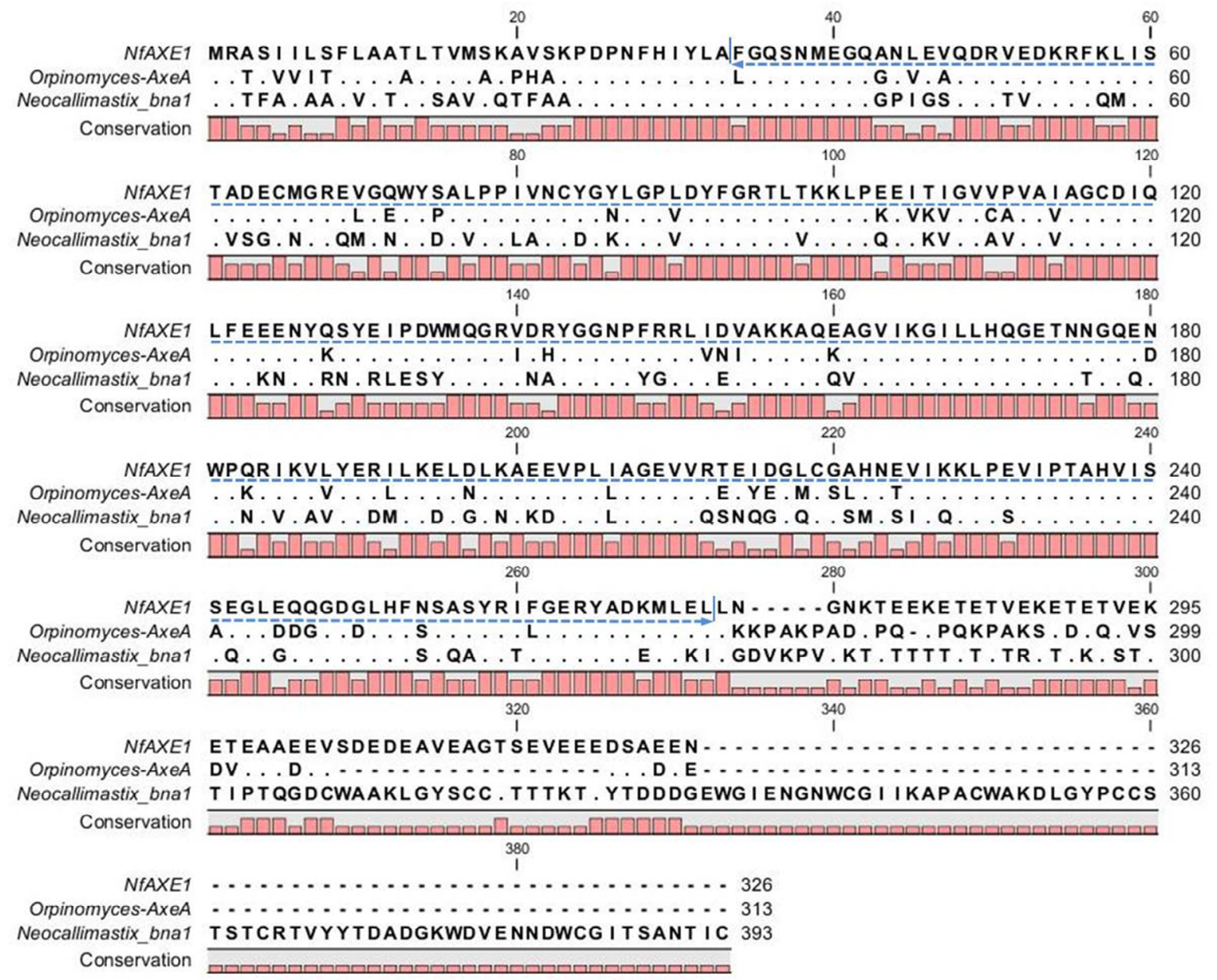

Figure 3. Comparison of deduced amino acid sequences of NfAXE1 with other anaerobic fungal acetyl xylanesterases (AXEs). AxeA, AXE from Orpinomyces sp. strain PC-2 (accession number AF001178); bnaI, AXE from Neocallimastix patriciarum (accession number U66251); NfAXE1, AXE from Neocallimastix frontalis PMA 02 (this report). Dotted arrow indicates regions for the conserved DUF 303. 


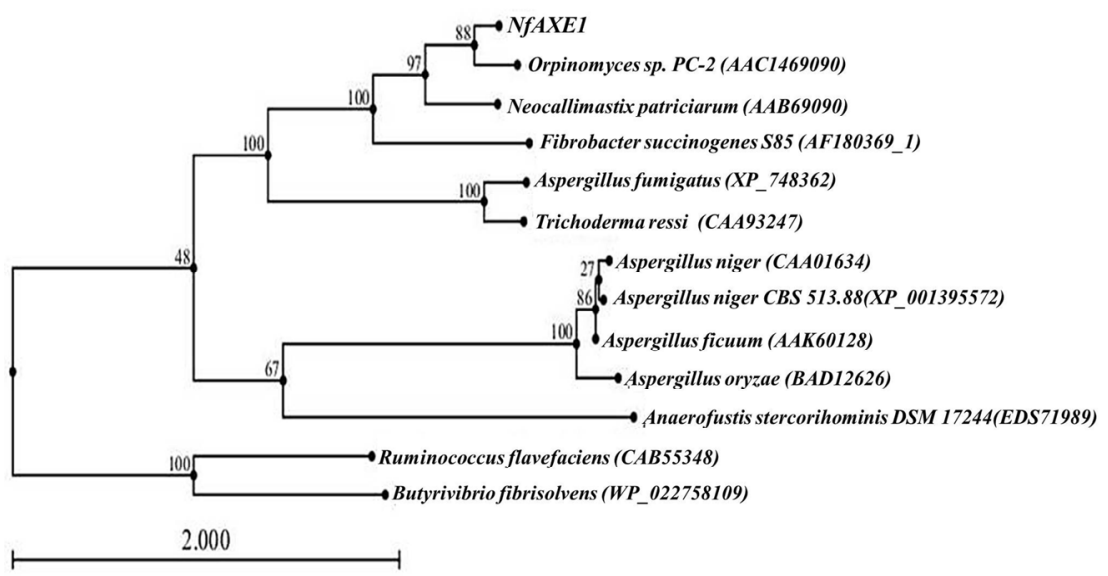

Figure 4. Phylogenetic analysis of NfAXE1 with known AXEs. Among the deduced AXE amino acid sequences deposited in the GenBank database, we selected amino acid sequences with $>40 \%$ identity with those of NfAXE1 for the phylogenetic analysis. The phylogenetic tree was built by the neighbor-joining method using the CLC Main Workbench program. GenBank accession numbers are given in parenthesis. NfAXE1, AXE from Neocallimastix frontalis PMA 02; AXE, acetyl xylanesterase.

(A)

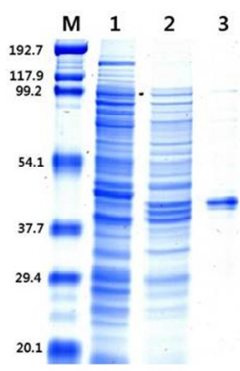

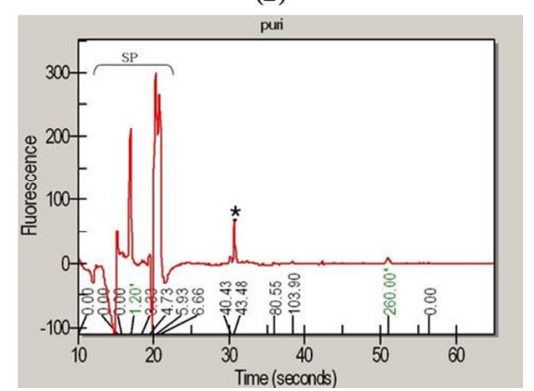

Figure 5. Protein purification of heterologously expressed NfAXE1 in Escherichia coli. (A) SDS-PAGE with Coomassie Blue staining of proteins at various stages of purification. M, Molecular markers $(\mathrm{kDa})$. Lanes: 1, Cells harboring pET28a as control; 2, crude cells harboring pET28a-NfAXE1 after IPTG induction; 3, purified recombinant NfAXE1. (B) SP, Internal standard signal peaks; *, purified target protein peak analyzed using the microfluidic system. NfAXE1, Neocallimastix frontalis PMA02 acetyl xylan esterase; SDS-PAGE, sodium dodecyl sulfate-polyacrylamide gel electrophoresis.

xylose released from acetylated birchwood xylan increased by 1.4-fold compared to the NfAXE1-free condition (Figure 7).

\section{DISCUSSION}

A gene encoding an NfAXE1 with a 981 bp ORF with no intron was isolated from the anaerobic rumen fungus $N$.

frontalis PMA02. The absence of an intron in the DNA sequence of this NfAXE1 suggests the potential for horizontal gene transfer from prokaryotes to anaerobic fungi in the rumen. This possibility is supported by a report of the horizontal gene transfer of glucose hydrolyzing enzymes from the rumen anaerobic bacteria Fibrobacter succinogens to the rumen anaerobic fungus Orpinomyces jouonii (GarciaVallvé et al., 2000).

A previously reported AXE in $N$. frontalis (AAQ10006.1) contains the XynB-like domain (cd01833) and CBM_10 domain (pfam02013; cellulose or protein binding domain), in contrast to NfAXE1, which contained only DUF303. Based on classification of the conserved domain of the enzyme, CEs can be classified into16 families, and AXEs (EC 3.1.1.72) can be found in eight of these CE families [1, 2, 3, 4, 5, 6, 7, 12 in the CAZy (Carbohydrate Active Enzyme; http://www.cazy.org) database]. AXEs containing DUF303, such as AxeA (AAC14690.1) from Orpinomyces sp. PC-2 (Blum et al., 1999) and BnaA (AAB69090.1) from $N$. patriciarum, have been placed in CE family 6 (Dalrymple et al., 1997). The only difference between AxeA and BnaA is the presence of CBM_10 (pfam02013; a cellulose or protein binding domain) in BnaA. The presence of DUF303 in NfAXE1 allows it to be classified into CE family 6 along with AxeA and BnaA. Interestingly, the anaerobic fungus $N$. patriciarum possesses not only CE family 6 AXE but also AXEs from $\mathrm{CE}$ families 1,2 , and 3 containing the

Table 1. Purification of NfAXE1 from Escherichi coli BL21 (DE3)

\begin{tabular}{lccccc}
\hline & $\begin{array}{c}\text { Total protein } \\
(\mathrm{mg})\end{array}$ & $\begin{array}{c}\text { Total activity } \\
\left(\text { units }^{1}\right)\end{array}$ & $\begin{array}{c}\text { Specific activity } \\
\text { (units/mg) }\end{array}$ & $\begin{array}{c}\text { Yield } \\
(\%)\end{array}$ & $\begin{array}{c}\text { Purification } \\
\text { (fold) }\end{array}$ \\
\hline Crude extact & 543.0 & 536.9 & 1.0 & 100 & 1.0 \\
Ni-NTA & 1.3 & 130.1 & 100.1 & 24.2 & 101.2 \\
\hline
\end{tabular}

NfAXE1, Neocallimastix frontalis PMA02 acetyl xylan esterase1.

${ }^{1}$ Activity was measured by determining the amount of $p$-nitrophenol formed from $p$-nitrophenyl acetate as substrate. 

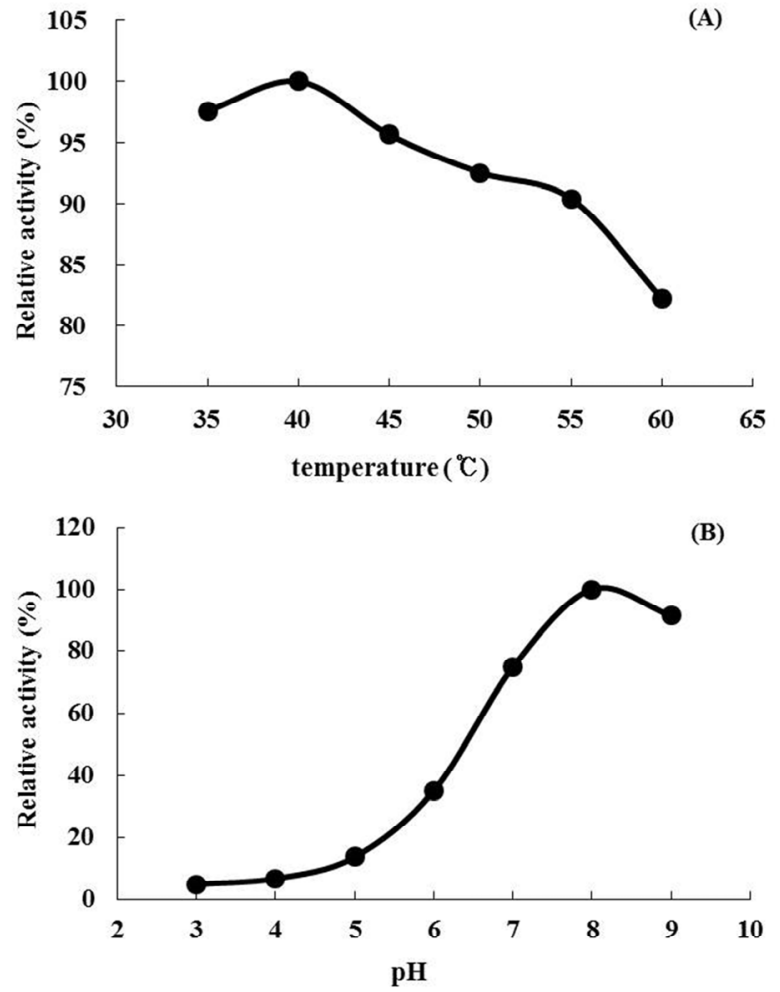

Figure 6. Effects of temperature (A) and $\mathrm{pH}(\mathrm{B})$ on the activity of recombinant NfAXE1 using $p$-nitrophenyl acetate as substrate. NfAXE1, Neocallimastix frontalis PMA02 acetyl xylan esterase1.

Peptidase_S9_superfamily (cl19141), Endoglucanase E-like (cd01831), and XynB-like (cd01833) domains, based on both the CAZy database and NCBI database, thereby suggesting the strong possible existence of AXEs from CE families 1,2, and 3 in $N$. frontalis. AXEs from anaerobic fungi have been detected in CE families containing AXEs from dominant rumen bacteria, raising the possibility that NfAXEs are present in CE families 4 and 12. However, to date, there is no solid evidence in the public databases supporting this possibility, and further research with modern molecular biology techniques is required.

Because the placement of a specific CE enzyme into the appropriate CE family is based on the identity of its conserved domain, any change at the level of an individual amino acid will not notably affect its classification. Consequently, the $\mathrm{pI}$ value of an AXE may not correlate with the CE family, but with the amino acid composition of the AXE. Compared to other AXEs identified in anaerobic fungi, NfAXE1 contains a higher proportion (63\%) of negative amino acids, which in turn results in the enzyme having are relatively lower pI value (4.54). Estimated pI values of AXEs from anaerobic fungus $N$. patirciarum range from 4.6 (BnaB) to 7.84 (BnaA). Therefore, it is possible that the AXEs present in $N$. frontalis differ from those present in $N$. patriciarum only in $\mathrm{pI}$ value, although this possibility requires further investigation.

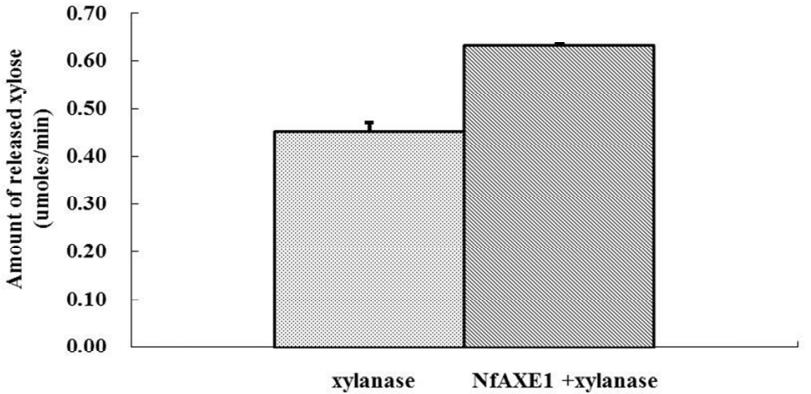

Figure 7. Synergistic effect of the xylanase and NfAXE1 mixture in terms of xylose release from acetylated birchwood xylan. xylanase, Trichoderma longibrachiatum; NfAXE1, Neocallimastix frontalis PMA02 acetyl xylan esterase1.

The low portion of sulfur-containing amino acids (3\%) in NfAXE1, accounting for the low number (10) of sulfur atoms embedded in the structure of the protein, could explain the absence of disulfide bonds in NfAXE1. Also, the higher instability index of NfAXE1 relative to those of other AXEs from anaerobic fungi demonstrates that the AXEs from other anaerobic fungi are more stable than NfAXE1. The standard definition of a stable protein is one with an instability index of $<40$ (Guruprasad et al., 1990). The thermostablility of a protein increases with increases in the aliphatic index. The aliphatic index of NfAXE1 (86.13) and AxeA (86.29) are higher than that of other AXEs from anaerobic fungi (Ikai, 1980). According to definition of Ikai (1980), the thermostability of both NfAXE1 and AxeA may be higher than that of other AXEs isolated from anaerobic fungi. However, despite its higher aliphatic index, the optimum temperature for AxeA activity (Blum et al., 1999) is lower than that for optimum NfAXE1 activity, although both show comparable thermostability. The optimum temperature of a hyperthermostable AXE from the hyperthermophillic bacterium Thermotoga maritime is $90^{\circ} \mathrm{C}$ (Drzewiecki et al., 2010), and the aliphatic index of this enzyme (73.51) is lower than that of NfAXE1. If the aliphatic index were to be linearly correlated with the temperature optimum and thermostability, the temperature optimum of NfAXE1 should be higher than $90^{\circ} \mathrm{C}$. From this result, we conclude that the aliphatic index may be only one of a number of indicators of protein stability.

The acetyl group at C-2 or C-3 of the xylose residues in the backbone is deacetylated by AXE, with the efficiency of

Table 2. Substrate specificity and specific activity of NfAXE1

\begin{tabular}{lcc}
\hline & $\begin{array}{c}\text { Specific activity } \\
\text { (units/mg) }\end{array}$ & $\begin{array}{c}\text { Relative activity } \\
\text { (\%) }\end{array}$ \\
\hline p-Nitrophenyl acetate & 100.1 & 100 \\
$\alpha$-Naphthyl acetate & 61.2 & 61.2 \\
Acetylated birchwood xylan & 56.3 & 56.3 \\
Acetylated beechwood xylan & 80.9 & 80.8 \\
\hline
\end{tabular}

NfAXE1, Neocallimastix frontalis PMA02 acetyl xylan esterase1. p-Nitrophenyl acetate was used as the standard. 
the enzymatic reaction increasing with decreasing length of the xylose backbone (Biely et al., 2013). This varying efficiency may explain our results on the substrate specificities of NfAXE1. The partially purified recombinant NfAXE1 displayed broad substrate specificity toward $p$-NA, $\alpha$-naphthyl acetate, acetylated birchwood xylan, and acetylated beechwood xylan, with the highest specific activity toward $p$-NA, which contains only one benzene ring. In comparison, the backbone of $\alpha$-naphthyl acetate contains two benzene rings, and acetylated natural xylan, including birchwood xylan and beechwood xylan, may have a long oligosaccharide backbone.

According to BRENDA (Braunschweig Enzyme Database; http://www.brenda-enzymes.org), the specific activity of XynS20E from $N$. patriciarum is higher than that of NfAXE1. However, comparisons of enzyme efficiency based only on values obtained under varying assay conditions with different substrates are not always meaningful. In addition, XynS20E is not a pure AXE, but a bifunctional xylanse, which may possibly explain its ability to release both reducing sugar and acetate from the substrate. In addition, it has been reported that the amount of reducing sugar liberated by xylanase could be increased by using AXE to remove the carboxyl group attached to xylan (Cybinski et al., 1999). We observed a synergistic effect of a xylanase and AXE cocktail on the release of reducing sugars, which is in agreement with the results of a previous study (Selig et al., 2008). These results suggest that the extent of acetylated hemicellulose degradation is limited by the acetylation of hemicellulose and that the addition of NfAXE1 to the reaction may increase the efficiency of xylanase for xylan degradation.

The rumen is maintained at $\mathrm{pH} 6$ to 7 under normal conditions, and the $\mathrm{pH}$ optimum of most rumen microbial enzymes falls within this range. However, the optimum $\mathrm{pH}$ of NfAXE1 was 8, which is similar to that of the AXE isolated from $N$. patriticum. It is therefore possible that any change in the protein expression system from Escherichia coli to the anaerobic fungus could affect the protein tertiary structure, thereby resulting in a change in the $\mathrm{pH}$ optimum. Further research is required to resolve this question.

\section{CONFLICT OF INTEREST}

We certify that there is no conflict of interest with any financial organization regarding the material discussed in the manuscript.

\section{ACKNOWLEDGMENTS}

This research was supported by Korea National Open University Research Fund.

\section{REFERENCES}

Altaner, C., B. Saake, M. Tenkanen, J. Eyzaguirre, C. B. Faulds, P. Biely, L. Viikari, M. Siika-aho, and J. Puls. 2003. Regioselective deacetylation of cellulose acetates by acetyl xylan esterases of different CE-families. J. Biotechnol. 105:95-104.

Biely, P. 1985. Microbial xylanolytic systems. Trends Biotechnol. 3:286-290.

Biely, P., M. Cziszárová, I. Uhliariková, J. W. Agger, X. L. Li, V. G. H. Eijsink, and B. Westereng. 2013. Mode of action of acetylxylan esterases on acetyl glucuronoxylan and acetylated oligosaccharides generated by a GH10 endoxylanase. Biochim. Biophys. Acta 1830:5075-5086.

Blum, D. L., X. L. Li, H. Chen, and L. G. Ljungdahl. 1999. Characterization of an acetyl xylan esterase from the anaerobic fungus Orpinomyces sp. strain PC-2. Appl. Environ. Microbiol. 65:3990-3995.

Bradford, M. M. 1976. A rapid and sensitive method for the quantitation of microgram quantities of protein utilizing the principle of protein-dye binding. Anal. Biochem. 72:248-254.

Coutinho, P. M. and B. Henrissat. 1999. Carbohydrate-active enzymes: an integrated database approach. In: Recent Advances in Carbohydrate Bioengineering (Eds. H. J. Gilbert, G. J. Davies, B. Henrissat, and B. Svensson). The Royal Society of Chemistry, Cambridge, UK. pp. 3-12.

Cybinski, D. H., I. Layton, J. B. Lowry, and B. P. Dalrymple.1999. An acetylxylan esterase and a xylanase expressed from genes cloned from the ruminal fungus Neocallimastix patriciarum act synergistically to degrade acetylated xylans. Appl. Microbiol. Biotechnol. 52:221-225.

Dalrymple, B. P., D. H. Cybinski, I. Layton, C. S. McSweeney, G. P. Xue, Y. J. Swadling, and J. B. Lowry.1997. Three Neocallimastix patriciarum esterases associated with the degradation of complex polysaccharides are members of a new family of hydrolases. Microbiology 143:2605-2614.

Drzewiecki, K., A. Angelov, M. Ballschmiter, K. J. Tiefenbach, R. Sterner, and W. Liebl. 2010. Hyperthermostable acetyl xylan esterase. Microb. Biotechnol. 3:84-92.

Fujino, Y., K. Ogata, T. Nagamine, and K. Ushida. 1998. Cloning, sequencing, and expression of an endoglucanase gene from the rumen anaerobic fungus Neocallimastix frontalis $\mathrm{MCH} 3$. Biosci. Biotechnol. Biochem. 62:1795-1798.

Garcia-Vallvé, S., A. Romeu, and J. Palau. 2000. Horizontal gene transfer of glycosyl hydrolases of the rumen fungi. Mol. Biol. Evol. 17:352-361.

Garcia-Campayo, V. and T. M. Wood. 1993. Purification and characterisation of a beta-D-xylosidase from the anaerobic rumen fungus Neocallimastix frontalis. Carbohydr. Res. 242:229-245.

Gasteiger, E., C. Hoogland, A. Gattiker, S. E. Duvaud, M. R. Wilkins, R. D. Appel, and A. Bairoch. 2005. Protein identification and analysis tools on the ExPASy server. In: The Proteomics Protocols Handbook (Ed. J. M. Walker). Humana Press, Totowa, NJ, USA. pp. 571-607.

Guruprasad, K, B. V. B. Reddy, and M. W. Pandit. 1990. Correlation between stability of a protein and its dipeptide composition: a novel approach for predicting in vivo stability of a protein from its primary sequence. Protein Eng. 4:155-161. 
Gutiérrez, R., E. Cederlund, L. Hjelmqvist, A. Peirano, F. Herrera, D. Ghosh, W. Duax, H. Jörnvall, and J. Eyzaguirre. 1998. Acetyl xylan esterase II from Penicillium purpurogenum is similar to an esterase from Trichoderma reesei but lacks a cellulose binding domain. FEBS Lett. 423:35-38.

Ho, Y. W., N. Abdullah, and S. Jalaludin. 1988. Penetrating structures of anaerobic rumen fungi in cattle and swamp buffalo. J. Gen. Microbiol. 134:177-181.

Ikai, A. 1980. Thermostability and aliphatic index of globular proteins. J. Biochem. 88:1895-1898.

Johnson, K. G., J. D. Fontana, and C. R. MacKenzie. 1988. Measurement of acetylxylan esterase in Streptomyces. Meth. Enzymol. 160:551-560.

Jue, C. K. and P. N. Lipke. 1985. Determination of reducing sugars in the nanomole range with tetrazolium blue. J. Biochem. Biophys. Methods 11:109-115.

Kwon, M., J. Song, J. K. Ha, H. Park, and J. Chang. 2009. Analysis of functional genes in carbohydrate metabolic pathway of anaerobic rumen fungus Neocallimastix frontalis PMA02. Asian Australas. J. Anim. Sci. 22:1555-1565.

Li, X. L. and R. E. Calza. 1991. Fractionation of cellulases from the ruminal fungus Neocallimastix frontalis EB188. Appl. Environ. Microbiol. 57:3331-3336.
McDermid, K. P., C. R. Mackenzie, and C. W. Forsberg. 1990. Esterase activities of Fibrobacter succinogenes subsp. succinogenes S85. Appl. Environ. Microbiol. 56:127-132.

Mountfort, D. O. and R. A. Asher. 1985. Production and regulation of cellulase by two strains of the rumen anaerobic fungus Neocallimastix frontalis. Appl. Environ. Microbiol. 49:13141322.

Orpin, C. G. 1975. Studies on the rumen flagellate Neocallimastix frontalis. J. Gen. Microbiol. 91:249-262.

Orpin, C. G. 1981. Isolation of cellulolytic Phycomycete fungi from the caecum of the horse. J. Gen. Microbiol. 123:287-296.

Selig, M. J., E. P. Knoshaug, W. S. Adney, M. E. Himmel, and S. R. Decker. 2008. Synergistic enhancement of cellobiohydrolase performance on pretreated corn stover by addition of xylanase and esterase activities. Bioresour. Technol. 99:4997-5005.

Tenkanen, M. 1998. Action of Trichoderma reesei and Aspergillus oryzae esterases in the deacetylation of hemicelluloses. Biotechnol. Appl. Biochem. 27:19-24.

Thomson, J. A. 1993. Molecular biology of xylan degradation. FEMS Microbiol. Rev. 104:65-82.

Wood, T. M., C. A. Wilson, and S. I. McCrae. 1995. The cellulase system of the anaerobic rumen fungus Neocallimastix frontalis: studies on the properties of fractions rich in endo- $(1 \rightarrow 4)$-betaD-glucanase activity. Appl. Microbiol. Biotechnol. 44:177-184. 